\title{
Systematic characterization of fluorophore behavior in the presence of electron microscopy sample preparation reagents.
}

Haiyan $\mathrm{Li}^{1}$, Danielle M. Jorgens ${ }^{1}$, Lei Wang ${ }^{2}$, Robert M. Stongin ${ }^{2}$, Joe W. Gray ${ }^{1,3,4}$ and Summer L. Gibbs ${ }^{1,3,4}$

1. Department of Biomedical Engineering, Oregon Health and Science University, Portland, OR 97201.

2. Department of Chemistry, Portland State University, Portland, OR 97201.

3. Knight Cancer Institute, Oregon Health and Science University, Portland, OR 97201.

4. OHSU Center for Spatial Systems Biomedicine, Oregon Health and Science University, Portland, OR 97201.

Correlative light and electron microscopy (CLEM) combines the strengths of multicolor fluorescence labeling utilizing light microscopy (LM) with the near angstrom resolution of electron microscopy (EM) making it an ideal tool to interrogate biology at the nanoscale. However, combining these two microscopy techniques is not a trivial task, as sample preparation for fluorescence LM is typically completed using hydrated samples that are thick by comparison to the samples used for EM. Additionally, sample preparation for EM involves dehydration, staining with heavy metal contrast, and embedding in plastic-like resin, which are typically thought to be incompatible with the low levels of fluorescent staining desirable for molecularly targeted applications. In an effort to expand and advance efforts toward direct CLEM experimentation, we sought to holistically characterize commonly used fluorophores as substrates for correlative microscopy.

In the current work, the sensitivity of four commonly used fluorophores including Atto 488, BODIPY FL, Cy3B, and Alexa Fluor 647 that represent three different fluorophore scaffolds, rhodamine, BODIPY, and cyanine, were thoroughly tested for their ability to withstand EM sample preparation. Each fluorophore was characterized in the presence of the typical chemicals used for EM sample preparation in such a way as to mimic both a benchtop environment (conventional) and also cryopreservation methods (high pressure freezing and freeze substitution). Fluorophores were characterized systematically through the addition of single EM staining reagents to determine the percentage of fluorescence decrease cased by each staining reagent before completing the entire EM sample preparation on fluorescently stained samples.

We spectrally characterized fluorophores in-solution and conjugated to bovine serum albumin (BSA) in the presence of heavy metal stains including osmium tetroxide and uranyl acetate at concentrations ranging from $0.03-4 \%$, as well as two common resins used for CLEM samples including Lowicryl HM20 and London Resin (LR) White over an eight hour time period. Some in-solution fluorescence decrease was seen in organic solvents, although no time dependence was observed pointing to solvent dependent fluorescence intensity rather than a time dependent interaction between the fluorophore and the solvent. Interestingly, LR White had a more detrimental affect over time on fluorescence intensity than Lowicryl HM20, a result that was utilized during our preparation of samples for CLEM studies. The same fluorophores were spectrally characterized conjugated to bovine serum albumin (BSA) to more closely mimic the environment within cells in the presence of varying concentration of heavy metals over time. 
To further elucidate the relationship between protein fluorophore binding and the effect of heavy metal staining, wheat germ agglutinin (WGA) was conjugated to each fluorophore and used to label cell membranes both in suspension as well as adhered upon cover glass. In both samples significantly more fluorescence was seen at all concentrations of uranyl acetate staining as compared to osmium tetroxide staining, an effect previously reported $(1,2)$. Cell membrane visualization using CLEM was completed as a proof of concept for future work that will utilize the fluorophore preservation strategies we have determined through this study.

\section{References}

[1] Peddie CJ, et al. (2014) Correlative and integrated light and electron microscopy of in-resin GFP fluorescence, used to localise diacylglycerol in mammalian cells. Ultramicroscopy 143:3-14. doi:10.1016/j.ultramic.2014.02.001 PMID:24637200

[2] Biel SS, Kawaschinski K, Wittern KP, Hintze U, \& Wepf R (2003) From tissue to cellular ultrastructure: closing the gap between micro- and nanostructural imaging. Journal of microscopy 212(Pt 1):91-99. doi:10.1046/j.1365-2818.2003.01227.x PMID:14516366 